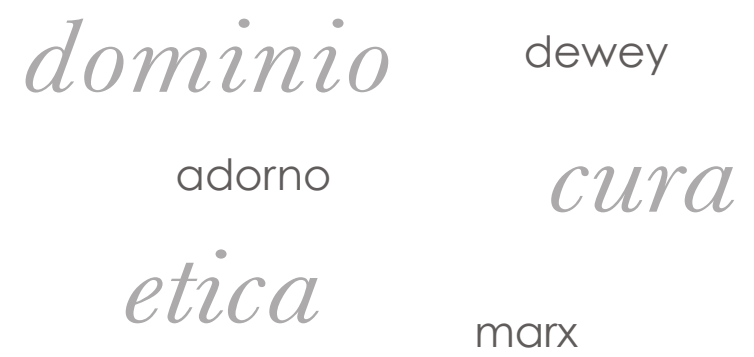

도을

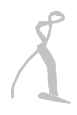

63

$2018 / 3$

FrancoAngeli 
Direttore: Ferruccio Andolfi.

Segreteria di redazione: Simona Del Bono, Donatella Gorreta, Sandra Manzi-Manzi.

Redazione: Silvano Allasia, Simona Bertolini, Valeria Bizzari, Thomas Casadei, Marco Deriu, Giulia Lasagni, Chiara Marchetti, Corrado Piroddi, Marina Savi, Mauro Simonazzi, Timothy Tambassi, Chiara Tortora.

Comitato editoriale: Nicola Antonetti (università di Parma), Laura Boella (università di Milano), Alessandro Bosi (università di Parma), Beatrice Centi (università di Parma), Paolo Costa (fondazione "Bruno Kessler" di Trento), Enrico Donaggio (università di Torino), Roberto Escobar (università di Milano), Alessandro Ferrara (università di Roma "Tor Vergata"), Rino Genovese (Scuola Normale di Pisa), Marco Ingrosso (università di Ferrara), Sergio Manghi (università di Parma), Elena Pulcini (università di Firenze), Loredana Sciolla (università di Torino), Alberto Siclari (università di Parma), Italo Testa (università di Parma), Silvia Vegetti Finzi (università di Pavia).

Comitato scientifico internazionale: Gabriel Amengual, Thomas Baumeister, Ronald Beiner, Fina Birulés, Nancy Fraser, Ágnes Heller, Axel Honneth, Charles Larmore, Steven Lukes, Emmanuel Renault, Jordi Riba, Fernando Savater, Jacques Texier, Carlos Thiebaut, Adriana Veríssimo Serrao, Maurice Weyemberg.

Consulenti e collaboratori: Angela Ales-Bello, Carlo Altini, Andrea Borsari, Francesca Brezzi, Rosa Calcaterra, Antonella Cutro, Annamaria Contini, Franco Crespi, Marcella D'Abbiero, Dimitri D’Andrea, Fulvia De Luise, Giuseppe Farinetti, Francesco Fistetti, Gregor Fitzi, Marisa Forcina, Vittoria Franco, Wolfgang Huemer, Antonino Laganà, Carmen Leccardi, Aldo Meccariello, Rita Messori, Stefano Petrucciani, Gianfranco Ragona, Annamaria Rivera, Fabio Rossi, Marco Solinas, Davide Sparti, Gabriella Turnaturi, Nadia Urbinati, Adriano Zamperini.

Tutti gli articoli inviati alla rivista sono valutati da referee anonimi

Direzione e redazione: Dipartimento di Filosofia - Via D'Azeglio 85 - 43100 Parma - Tel. 0521-902340. Fax: 902551. e-mail: ginestra@unipr.it

Sito: www.laginestra.unipr.it

con il contributo della:

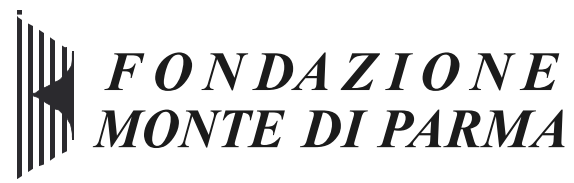

Progetto grafico: Rossana Capasso. 


\section{SOMMARIO}

\section{DEWEY E LA FILOSOFIA SOCIALE}

7 Rosa M. Calcaterra

Etica, morale, politica: proposte deweyane

20 Federica Gregoratto

Critica del dominio di genere e di classe

Verso una lettura intersezionalista di J. Dewey e J. Addams

$35 \quad$ Corrado Piroddi

Conflitto, riconoscimento e dominio

Tra John Dewey e Pierre Bourdieu

$47 \quad$ Matteo Santarelli

Conflitto, interessi, bisogni

Le Lezioni in Cina di John Dewey

\section{ETICA E POLITICA}

a cura di Marco Solinas

61 Dimitri D'Andrea

Uno strano realismo politico

Max Weber e l'etica

$77 \quad$ Anna Loretoni

Etica della cura e vulnerabilità degli individui

$91 \quad$ Marco Solinas

La normatività di Marx

Critica immanente, etica e politica 


\section{ARCHIVIO}

107 Giovanni Sgro'

Alle origini della «Nuova Lettura di Marx»

Backhaus su Adorno e Marx

109 Hans-Georg Backhaus

Theodor W. Adorno su Marx

e sui concetti fondamentali della teoria sociologica

\section{DOMINIO E SCHIAVITÙ}

123 Italo Testa

Vita e dominio

Servo-padrone, assoggettamento, riconoscimento

140 Thomas Casadei

Corpi in transito

Sulla tratta contemporanea

\section{NOTE DI LETTURA}

157 Fulvia de Luise, a cura di, Cittadinanza. Inclusi ed esclusi tra gli antichi e i moderni (Federico Zuolo); Martin Buber, Antica e nuova comunità (Francesco Ferrari); Donatella Di Cesare, Stranieri residenti. Una filosofia dell'emigrazione (Chiara Tortora); Karl Marx, Manoscritti economico-filosofici del 1844, a cura di F. Andolfi e G. Sgro' (Francesca Sofia Alexandratos)

170 AUTORI 
etica e politica

\title{
La normatività di Marx \\ Critica immanente, etica e politica
}

Marco Solinas*

\begin{abstract}
The paper aims to provide a critical analysis of Marx's normative conception, both in terms of his economicistc mistakes and in relation to the normative principles implied in his general theoretical framework. The attention is then focused on Marx's immanent critique of capitalism, also in relation to the normative interpretation of socialism recently presented by Axel Honneth; the Author highlights as Marx's concept of freedom is linked to the idea of liberation from forced labour.

KEYWORDS: Normativity, capitalism, Karl Marx, immanent critique, Axel Honneth, freedom.
\end{abstract}

Una critica del capitalismo libera da ogni determinismo storico, e pertanto consapevolmente carica sul piano normativo, può attingere al lascito marxiano? Certo è che Marx ha compiuto l'errore fatale di sottostimare, neutralizzare e talvolta persino azzerare ruolo e funzioni di una molteplicità di elementi e piani normativi costitutivi delle società moderne, a tutto vantaggio della sfera economica, intesa quale «struttura» rispetto alla quale l'intera dimensione del diritto e dello Stato, nonché della cultura, della famiglia e della scienza, è stata presentata come poco più che un riflesso ideologico «sovrastrutturale» dei rapporti di produzione vigenti - sebbene $\mathrm{i}$ progressi normativi acquisiti sul piano giuridico-politico non siano stati totalmente disconosciuti da Marx, ma piuttosto circoscritti e depotenziati. Coniugando tale economicismo a una filosofia della storia rigidamente deterministica, le cui ferree leggi avrebbero dovuto condurre necessariamente il proletariato a costituirsi in una classe politicamente consapevole di sé e a rovesciare il sistema capitalistico, Marx è stato indotto ad accorciare drasticamente anche il raggio della critica sociale, appiattendola sul piano della «presa di coscienza» e della «accelerazione» di quanto sarebbe comunque dovuto avvenire - seppur sempre con una certa tensione: ma come e quando, di preciso? L'auto-interpretazione di Marx della sua stessa opera intellettuale, sul doppio piano scientifico e politico, è stata in tal senso segnata da quello che definirei come un radicale auto-fraintendimento scientista-deterministico volto a negarne (platealmente) ogni tipo di normatività:

Articolo presentato il 2 ottobre 2018, accettato il 28 ottobre 2018. Affiliazione: Scuola Superiore Sant'Anna di Pisa. Indirizzo email: marco.solinas@santannapisa.it.

La società degli individui, n. 63, anno XXI, 2018/3 ISSN 1590-7031, ISSNe 1972-5752 
Le proposizioni teoriche dei comunisti non si basano affatto su idee, su principi inventati o scoperti da questo o quell'individuo che vuole migliorare il mondo. Sono soltanto espressioni generali di rapporti effettivi di una lotta di classe che esiste, di un movimento storico che si svolge sotto i nostri occhi ${ }^{1}$.

Se non fosse, appunto, che questa audace asserzione venne affidata al Manifesto del partito comunista, dunque a un mezzo deputato a contribuire a cambiare il mondo, anzi a migliorarlo, mostrando e divulgando alcune delle idee e dei principi delle dinamiche storiche, sociali e politiche che $\mathrm{i}$ due autori ritennero di avere non tanto inventato, quanto piuttosto scoperto.

In termini più sistematici: di fronte ai deficit inerenti al piano normativo indotti da economicismo e determinismo, così come rispetto alla correlata auto-interpretazione anti-normativista, il pensiero di Marx può o non può contribuire a elaborare una critica del capitalismo che oggi può soltanto essere, consapevolmente, carica di significato normativo? Una via che mi pare interessante intraprendere per iniziare a rispondere a questa domanda è stata recentemente battuta da Axel Honneth nel volume L'idea di sociali$s m o^{2}$. Sottolineate con decisione le «tare congenite» economicistiche dell'impianto socialista tradizionale e marxiano, Honneth ha messo in luce come tale framework fosse ancorato fin dalla sua nascita ai principi di libertà, uguaglianza e fraternità che erano stati sanciti e legittimati dalla Rivoluzione francese; principi che fornirono un fondamentale puntello normativo alla critica rivolta dai socialisti e da Marx alla loro applicazione formale, e non sostanziale, all'interno dell'ordinamento capitalistico. Si tratta pertanto del ricorso a una normatività immanente, non più solo idealistico-trascendente e utopistica, nel senso che i principi guida cui si richiama Marx più o meno implicitamente sono incarnati nelle istituzioni giuridiche e politiche e negli orientamenti spirituali e culturali dell'ormai affermata società borghese post-rivoluzionaria. Nel presente contributo riprenderò questa prospettiva, e sosterrò così che nonostante Marx abbia esplicitamente negato di aver elaborato una teoria (normativa) volta a «migliorare il mondo», il suo framework teorico è invero normativamente carico; e sottolineerò che precisamente grazie a questo carico egli ha potuto elaborare una critica immanente del capitalismo che mantiene una certa validità a dispetto, e quindi oltre, $\mathrm{i}$ limiti posti dal suo economicismo e determinismo storico.

K. Marx, F. Engels, Manifesto del partito comunista, Feltrinelli, Milano 2017, p. 25; cfr. anche Iid., L'ideologia tedesca, Editori Riuniti, Roma 1958, p. 25: «Il comunismo per noi non è stato di cose che debba essere instaurato, un ideale al quale la realtà dovrà conformarsi. Chiamiamo comunismo il movimento reale che abolisce lo stato di cose presente. Le condizioni di questo movimento risultano dal presupposto ora esistente».

2 A. Honneth, L'idea di socialismo (2015), Feltrinelli, Milano 2016. 
Procedendo lungo questa via, mi discosterò tuttavia dalla tesi di Honneth secondo cui il fulcro dell'impianto normativo socialista e marxiano sarebbe stato rappresentato dal concetto di «libertà sociale», intesa anzitutto quale conciliazione dei principi di libertà e fraternità. Una prospettiva che mirando a compensare la precedente deriva ricostruttiva indotta dal radicale immanentismo neo-hegeliano del Diritto della libert $\grave{a}^{3}$, mi pare questa volta condurre Honneth a sbilanciarsi sul versante speculare, positivo, propositivo e persino costruttivo dell'agire etico solidale. Visuale che però lo induce a trascurare il ruolo che Marx attribuisce al principio normativo dell'uguaglianza, soprattutto nella forma negativa della critica immanente delle disuguaglianze costitutive del sistema di produzione capitalistico, nonché della critica della libertà formale liberale; così come lo spinge a marginalizzare ulteriormente l'analisi degli interessi antagonisti che attraversano la società civile, riflettendosi sul piano delle lotte politiche ${ }^{4}$. Tre elementi che credo rappresentino invece, una volta abbandonato il framework di sfondo, alcuni dei lasciti più significativi dell'opera di Marx, o perlomeno è quanto cercherò ora di mostrare.

Al fine di far emergere gli elementi normativi della critica marxiana ancor oggi fruibili, procederò presentando sinteticamente, in un primo passo, le falle del determinismo economicista, tali da rendere necessaria una revisione dell'impianto teorico complessivo anche sul piano normativo. In un secondo passo, presenterò alcuni dei punti salienti della normatività del framework marxiano sia rispetto alla delineazione della società comunista sia, soprattutto, rispetto alla tematizzazione della libertà quale forma di liberazione dal giogo del lavoro coercitivo salariato; concentrandomi così sulla sua critica immanente del capitalismo. In un ultimo passo proverò, infine, a spiegare meglio perché la concezione positiva normativa della libertà sociale improntata sul modello dell'etica della cura rilanciata da Honneth mi paia meno significativa della suddetta forma di critica immanente, anche in relazione all'analisi politico-economica della categoria di interesse, ai fini di un'attualizzazione dell'eredità marxiana.

Si veda la stessa dichiarazione d'apertura di Honneth, in op. cit., p. 10; il riferimento è anzitutto ad A Honneth, Il diritto della libertà. Lineamenti per un'eticità democratica (2011), Codice, Torino 2015; prospettiva neo-hegeliana che era stata varata con rigore già in A. Honneth, Il dolore dell'indeterminato. Un'attualizzazione della filosofia politica di Hegel (2001), manifestolibri, Roma 2013.

4 Ho provato a mettere in luce il deficit di politicità della prospettiva neo-hegeliana varata da Honneth, in M. Solinas, Morale, etica, politica. Sulla svolta neo-hegeliana della teoria critica tedesca, "Teoria politica", VIII, 2018, pp. 365-383. 


\section{Normatività negata}

La marginalizzazione e neutralizzazione, da parte di Marx, della sfera normativa inerente all'attività «sovrastrutturale» della critica sociale, e più in generale dei principi normativi quali elementi di legittimazione o messa in discussione dell'ordinamento sociale, trovano una giustificazione anzitutto nella sua filosofia della storia, tale da attribuire al proletariato pressoché ogni ruolo e funzione socialmente emancipatoria.

Individuato quale motore del divenire storico della modernità la lotta di classe tra proletariato e borghesia, Marx colloca tale contrapposizione entro un framework dialettico di matrice hegeliana stante il quale l'antagonismo tra le due classi si delinea come contraddizione generata in modo automatico e necessario dallo sviluppo storico della società civile medesima: «malgrado l'eccesso di ricchezza, la società civile non è ricca abbastanza, cioè nelle risorse ad essa peculiari non possiede abbastanza per ovviare all'eccesso della povertà e alla produzione della plebe» ${ }^{5}$; una contraddizione da intendersi - spiega sempre Hegel nella Filosofia del diritto - in termini dialettici:

Da questa sua dialettica la società civile viene spinta oltre sé, anzitutto questa determinata società, per cercare consumatori e con ciò i necessari mezzi di sussistenza fuori di essa in altri popoli, che le stanno addietro nei mezzi dei quali essa ha eccedenza, $o$ in genere nell'industria ecc. ${ }^{6}$.

Ove Hegel aveva preliminarmente chiarito trattarsi del rapporto fra una classe più ricca che accumula e «concentra» la ricchezza, e di una più povera, sulla quale ricade il peso del lavoro, e che non può pertanto godere «delle libertà» connesse all'incremento della ricchezza, e che tende a ingrandirsi e a decadere sempre più ${ }^{7}$. Marx riprende tale schema concettuale fin dai suoi primi scritti, come emerge limpidamente, per esempio, nella Miseria della filosofia:

Di giorno in giorno diventa dunque sempre più chiaro che i rapporti di produzione, entro i quali si muove la borghesia, non hanno un carattere unico, semplice, bensì un carattere duplice; che negli stessi rapporti entro i quali si produce la ricchezza, si produce altresì la miseria; che entro gli stessi rapporti nei quali si ha sviluppo di forze produttive, si sviluppa anche una forza produttrice di repressione;

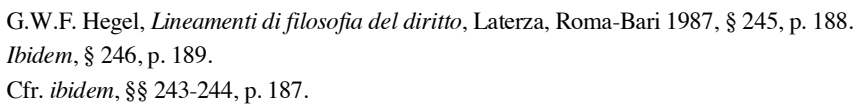


che questi rapporti producono la ricchezza borghese, ossia la ricchezza della classe borghese, solo a patto di annientare continuamente la ricchezza dei membri che integrano questa classe, e a patto di dar vita a un proletariato ognora crescente ${ }^{8}$.

Fondamentale, per Marx, è che questo proletariato ognora crescente verrà poi a trovarsi in una condizione sempre più disperata, ancor peggiore di quella della servitù della gleba, perché non avrà alcuna chance per migliorare le proprie condizioni di vita: lo sviluppo del sistema capitalistico è tale da condurre non a un miglioramento, ma a un peggioramento delle condizioni di vita dei proletari: li fa sprofondare vieppiù nella fame e nella miseria più nera ${ }^{9}$. Presupposta tale impossibilità sistematica di ascesa socio-economica, e presagitone anzi il futuro declino, i proletari verranno pertanto a trovarsi in una posizione di «antagonismo diretto» (direkten Gegensatz) rispetto allo Stato, nel senso che «per affermarsi personalmente» non avranno altra via che quella di rovesciare l'ordinamento capitalistico ${ }^{10}$. Antagonismo esacerbato dalla sovrappopolazione operaia generata dalla stessa dinamica dell'espansione capitalistica, come Marx scriverà molto più tardi nel Capitale:

Quanto maggiori sono la ricchezza sociale, il capitale in funzione, il volume e l'energia del suo aumento, quindi anche la grandezza assoluta del proletariato e la forza produttiva del suo lavoro, tanto maggiore è l'esercito industriale di riserva. [...] Ma quanto maggiore sarà questo esercito di riserva in rapporto all'esercito operaio attivo, tanto più in massa si consoliderà la sovrappopolazione la cui miseria è in proporzione inversa al tormento del suo lavoro ${ }^{11}$.

Più in breve, «nella misura in cui il capitale si accumula, la situazione dell'operaio, qualunque sia la sua retribuzione, alta o bassa, deve peggiorare» ${ }^{12}$; e si tratta di un peggioramento non solo inevitabile, ma quanto mai brutale:

Questa legge determina un'accumulazione di miseria proporzionata all'accumulazione di capitale. L'accumulazione di ricchezza all'uno dei poli è dunque al tempo stesso accumulazione di miseria, tormento di lavoro, schiavitù, ignoranza,

\footnotetext{
K. Marx, Miseria della filosofia, Rinascita, Roma 1949, p. 100; cfr. anche Id., Critica al programma di Gotha, Editori Riuniti, Roma 1990, p. 10

9 Cfr. K. Marx, F. Engels, Manifesto, cit., p. 22; Iid., L'ideologia tedesca, cit., p. 56; K. Marx, Il capitale, Editori Riuniti, Roma 1970, libro I, VII, cap. 23, p. 97

${ }^{10}$ K. Marx, F. Engels, L'ideologia tedesca, cit., p. 56.

${ }^{11}$ K. Marx, Il capitale, cit., libro I, VII, cap. 23, p. $95 \mathrm{sg}$.

12 Ibidem, p. 97.
} 
brutalizzazione e degradazione morale al polo opposto, ossia dalla parte che produce il proprio prodotto come capitale ${ }^{13}$.

Come si vede, lungo il corso della sua intera produzione scientifica, Marx tiene ferma la tesi dell'antagonismo dialettico tra borghesia e proletariato che già Hegel aveva chiaramente delineato nella Filosofia del diritto.

A differenza della soluzione proposta da Hegel, Marx 'individua' però la risoluzione della contraddizione inerente all'antagonismo di classe nella rivoluzione proletaria. Rivolgimento collocato saldamente entro una filosofia della storia rigidamente deterministica:

Le condizioni economiche avevano dapprima trasformato la massa della popolazione del paese in lavoratori. La dominazione del capitale ha creato a questa massa una situazione comune, interessi comuni. Così questa massa è già una classe nei confronti del capitale, ma non ancora per se stessa. Nella lotta, della quale abbiamo segnalato solo alcune fasi, questa massa si riunisce, si costituisce in classe per se stessa. Gli interessi che essa difende diventano interessi di classe. Ma la lotta di classe contro classe è una lotta politica ${ }^{14}$.

Il proletariato industriale, dunque, è destinato a guidare il movimento rivoluzionario, nel quale confluiranno i lavoratori esclusi dalla grande industria e i ceti medi, anch'essi destinati a incorrere - come Marx ribadirà a più riprese - in un inevitabile peggioramento delle proprie condizioni di vi$\mathrm{ta}^{15}$. Come recita, in sintesi, il Manifesto: «Con lo sviluppo della grande industria viene dunque sottratto da sotto i piedi della borghesia il terreno stesso su cui produce e si appropria dei prodotti. Essa produce anzitutto il suo proprio becchino. Il suo tramonto e la vittoria del proletariato sono ugualmente inevitabili» ${ }^{16}$.

Ora, sappiamo bene che la storia del capitalismo da Marx fino ai nostri giorni ha in larga parte falsificato tale diagnosi temporale nel suo snodo economico-politico forse più rilevante in assoluto: anziché ad una crescente proletarizzazione della borghesia, si è piuttosto assistito ad una progressiva borghesizzazione del proletariato. Più in generale, anziché una plebe affamata, l'economia capitalistica novecentesca è riuscita a fornire una quantità di beni e servizi di massa tali da innalzare il tenore di vita anche dei più poveri a dei livelli che erano certo del tutto impensabili a metà del XIX secolo

\footnotetext{
13 Ibidem.

${ }^{14}$ K. Marx, Miseria della filosofia, cit., p. 139.

15 Cfr., per esempio, K. Marx, F. Engels, L'ideologia tedesca, cit., p. 51; Iid., Manifesto, cit., pp. 17, 20; K. Marx, Critica al programma di Gotha, cit., p. 20

${ }^{16}$ K. Marx, F. Engels, Manifesto, cit., p. 23
} 
- e attualmente nei Paesi più poveri la ricchezza, seppur distribuita in modo diseguale, va crescendo a ritmi esponenziali. Viceversa, nei periodi di crisi, come l'attuale, nei Paesi più progrediti si assiste anche a fenomeni di parziale proletarizzazione della borghesia, esasperati nel contempo dall'incipiente demolizione del diritto del lavoro e del welfare state $^{17}$. E così, se tali dinamiche non hanno condotto alle condizioni preconizzate da Marx, peraltro anche in virtù delle lotte condotte (e vinte) in passato dai movimenti socialisti e comunisti, si assiste nondimeno a lacerazioni e conflitti socio-economici nei quali i tassi di disoccupazione, povertà e ricchezza relativi delle società prese di volta in volta in considerazione, e quindi dei rispettivi antagonismi, giocano un ruolo decisivo. Da questa prospettiva, alcuni degli strumenti critici elaborati da Marx, estrapolati dal quadro teorico totalizzante e deterministico in cui sono collocati originariamente, mostrano di essere ancor oggi assai affilati: sono preziosi, direi irrinunciabili.

La ripresa e l'aggiornamento critico di tali strumenti, però, deve far fronte anche al tramonto della tesi di sociologia politica stante la quale il proletariato a un certo punto sarebbe arrivato da sé a «prendere coscienza» dei propri interessi «obiettivi», in virtù dello sviluppo (necessario) delle forze di produzione. Così come si deve abbandonare la tesi correlata di teoria politica, secondo cui gli scienziati (sociali), a differenza dei teorici (utopisti), «devono solo rendersi conto di ciò che si svolge davanti ai loro occhi e farsene portavoce ${ }^{18}$. Tutto al contrario, abbandonato lo schematismo deterministico della filosofia della storia marxiana, diventa prioritaria la tematizzazione e soprattutto la problematizzazione di quei processi culturali e politici di ampio respiro attraverso i quali vengono costruite e stabilite socialmente le categorie che stabiliscono cosa e come deve, o non deve, essere considerato come «interesse» dei differenti gruppi sociali in gioco. Processi che non si lasciano certo ridurre a meri riflessi sovrastrutturali dei rapporti di produzione ${ }^{19}$ - come già all'inizio del XX secolo era stato segnalato con la massima decisione da autori quali Gramsci, Lukács, e dagli esponenti della teoria critica tedesca di fronte all'ascesa dei fascismi -, sebbene la dimensione economica continui a rappresentare uno degli aspetti fondamentali della costruzione sociale di tali categorie. Così come si deve ripensare interamente la questione delle forme della critica sociale, procedendo decisi nella stessa chiarificazione dell'auto-fraintendimento scientista anti-

\footnotetext{
17 Cfr., per esempio, L. Gallino, Il lavoro non è una merce. Contro la flessibilità, Laterza, Roma-Bari 2007.

${ }_{18}$ K. Marx, Miseria della filosofia, cit., p. 102.

19 Sulla fondamentale distinzione fra struttura e sovrastruttura cfr., per esempio, K. Marx, Per la critica dell'economia politica, Newton Compton, Roma 1972, pp. 30-31; ma si veda già Id., Manoscritti economico-filosofici del 1844, Einaudi, Torino 1968, p. 112.
} 
normativista di Marx. Infine, richiede una altrettanto radicale operazione di revisione la visione marxiana del politico, ridotto a mero specchio dei rapporti di produzione, come emerge senza veli dalla tesi secondo cui «con l'abolizione delle differenze di classe, scompaiono da sé tutte le disuguaglianze sociali e politiche che ne derivano» ${ }^{20}$. Una lettura che attesta l'indebita neutralizzazione della portata emancipatoria delle forme statuali democratiche, a tutto vantaggio dell'economico, la cui ricezione è stata peraltro nefasta per una parte consistente della tradizione comunista.

\section{Normatività e critica immanente}

La radicale marginalizzazione dei significati e valori normativi inerenti alle sfere culturali, giuridiche e politiche delle società moderne, nonché l'auto-interpretazione scientista e anti-normativista adottata da Marx rispetto alla sua propria diagnosi e prognosi socio-politica, non significa tuttavia che il suo framework teorico non riposi su una serie di fondamentali principi e assunzioni normative; al contrario: tali principi sono costitutivi della sua critica del capitalismo, ma sono presenti in forma perlopiù implicita.

In tal senso, è utile riprendere la tesi avanzata da Honneth quando scrive:

il socialismo rimanda fin dalle sue origini a un movimento di critica immanente del moderno orientamento sociale di tipo capitalistico. Di quest'ultimo vengono sì accettati i fondamenti normativi ancorati ai principi di libertà, uguaglianza e fraternità che lo legittimano, ma viene messo in dubbio che essi possano essere realizzati in modo non contraddittorio se la libertà non viene ripensata in senso meno individualistico, e dunque insistendo con maggior decisione in direzione di una sua applicazione di taglio intersoggettivo ${ }^{21}$.

Rispetto a questa lettura, condivido pienamente il richiamo alla fondamentale dimensione normativa che fornisce l'ossatura teorica della critica immanente svolta da Marx delle accezioni liberali, quindi 'solo' formali e non sostanziali, dei principi di libertà e uguaglianza. Viceversa, trovo decisamente meno convincente puntare sulla dimensione positiva dell'ideale di «libertà sociale», che avrebbe rappresentato la conciliazione e il supera-

\footnotetext{
${ }^{20}$ K. Marx, Critica al programma di Gotha, cit., p. 26; cfr. già Id., Miseria della filosofia, cit., p. 140: «La classe lavoratrice sostituirà, nel corso del suo sviluppo, all'antica società civile una associazione che escluderà le classi e il loro antagonismo, e non vi sarà più potere politico propriamente detto, poiché il potere politico è precisamente il riassunto ufficiale dell'antagonismo nella società civile [puisque le pouvoir politique est précisément le résumé officiel de l'antagonisme dans la société civile]».

21 A. Honneth, L'idea di socialismo, cit., p. 26 s.
} 
mento delle «contraddizioni» fra i tre principi cardinali della Rivoluzione, grazie a una modalità etica dell'agire l'uno-per-l'altro di alto profilo morale.

Certo in Marx si trova l'esplicita tematizzazione di una forma di comunità, quella comunista, nella quale l'accomunazione economica e la cooperazione rappresentano, in positivo appunto, l'applicazione di un principio morale di alto profilo e di un modello di comunità alternativo all'atomismo dell'ordinamento liberale; come leggiamo per esempio nel Manifesto: «Al posto della vecchia società borghese con le sue classi e le sue contrapposizioni di classe, subentra un'associazione dove il libero sviluppo di ciascuno è la condizione per il libero sviluppo di tutti» ${ }^{22}$. E ancora, a differenza dell'uguaglianza del diritto liberale, in questa comunità si applicherà un criterio di uguaglianza di più alto profilo, attento alle necessità e qualità di ognuno, tale per cui verrà fatto valere il principio, ribadito a chiare lettere nella Critica al programma di Gotha: «Ognuno secondo le sue capacità; a ognuno secondo i suoi bisogni» ${ }^{23}$. Il concetto di libertà viene del resto delineato da Marx, come leggiamo per esempio in La questione ebraica, alla luce di un ideale di cooperazione per cui l'altro non viene più percepito come un limite, ma piuttosto come una via per la propria autorealizzazione:

Il diritto dell'uomo alla proprietà privata è dunque il diritto di godere a proprio arbitrio (a son gré), senza considerare gli altri uomini, indipendentemente dalla società, del proprio patrimonio e di disporre di esso, il diritto dell'egoismo. Quella libertà individuale, come questa utilizzazione della medesima, costituiscono il fondamento della società civile. Essa lascia che ogni uomo trovi nell'altro uomo non già la propria realizzazione, ma piuttosto il limite della sua libertà ${ }^{24}$.

E tuttavia, anche qui, persino quando Marx ritrae a tinte fosche la figura dell' «uomo egoista» in quanto distinto dalla «persona morale» e quale fondamento della dissoluzione del «mondo degli uomini in un mondo di individui atomistici, ostilmente contrapposti gli uni agli altri» ${ }^{25}$, resta sempre centrale la dimensione negativa della critica: il modello positivo della società comunista si staglia quale forma speculare della società civile borghese, quale prodotto della sua negazione. Siamo così immediatamente ricondotti alla priorità attribuita alla sfera economica e ai suoi antagonismi, nella fattispecie alla critica della fondamentale dicotomia fra il cittadino-citoyen, portatore di diritti formali il cui grado effettivo di emancipazione resta per

\footnotetext{
${ }^{22}$ K. Marx, F. Engels, Manifesto del partito comunista, cit., p. 35.

23 K. Marx, Critica al programma di Gotha, cit., p. 16.

${ }^{24}$ K. Marx, La questione ebraica, manifestolibri, Roma, 2004, p. 194.

${ }^{25}$ Ibidem, p. 205.
} 
Marx inevitabilmente basso, poiché non incide sul piano economico dei rapporti di produzione, e il borghese-bourgeois, portatore di quegli interessi privati che determinano la reale oppressione della classe proletaria, la sua non libertà.

È su questo piano che si gioca la partita della critica immanente dell'ideologia dell'ordinamento giuridico liberale, che offre al proletariato una libertà soltanto immaginaria: «sotto il dominio della borghesia gli individui sono più liberi di prima, nell'immaginazione, perché per loro le loro condizioni di vita sono casuali; nella realtà sono naturalmente meno liberi perché più subordinati a una forza oggettiva ${ }^{26}$. Di contro - premette Marx - a essere «libero» è solo chi può sfuggire al gioco del lavoro alienato:

Solo nella comunità con altri ciascun individuo ha i mezzi per sviluppare in tutti i sensi le sue disposizioni; solo nella comunità diventa dunque possibile la libertà personale. Nei surrogati di comunità che ci sono stati finora, nello Stato ecc., la libertà personale esisteva soltanto per gli individui che si erano sviluppati nelle condizioni della classe dominante e solo in quanto erano individui di questa classe ${ }^{27}$.

Come si vede, la delineazione dell'alternativa positiva si staglia sempre a partire dal negativo, dal superamento dell'oppressione esistente.

Il principio normativo della libertà adottato da Marx nella sua critica immanente dell'ordinamento capitalistico è stato del resto correlato sistematicamente all'antagonismo costitutivo tra sfruttatori (oziosi) e sfruttati (incatenati) - posto a monte che «il sistema del lavoro salariato è un sistema di schiavitù» ${ }^{28}$ - lungo l'intera traiettoria del suo percorso teorico. Nei Manoscritti, per esempio, leggiamo che gli operai, «quanto più vogliono guadagnare, tanto più debbono sacrificare il loro tempo e privandosi completamente di ogni libertà compiere un lavoro da schiavi al servizio della avidità altrui» ${ }^{29}$; una diseguaglianza sostanziale, intesa anche quale «godimento ozioso dell'altrui sudore di sangue ${ }^{30}$, che trova una soluzione radicale nella soppressione della proprietà privata, quindi nel ristabilimento di una uguaglianza (Gleichheit) che permette lavoro libero (freie Arbeit) e libero godimento (freien Genu $\beta$ ) a tutti ${ }^{31}$. Questa idea fondamentale della libertà

\footnotetext{
${ }^{26}$ K. Marx, F. Engels, L'ideologia tedesca, cit., p. 55.

27 Ibidem, pp. 54-55.

${ }^{28}$ K. Marx, Critica al programma di Gotha, cit., p. 25; cfr. anche ibidem, p. 8: «l'uomo, il quale non ha altra proprietà all'infuori della sua forza-lavoro, deve essere, in tutte le condizioni di società e di civiltà, lo schiavo degli altri uomini che si sono resi proprietari delle condizioni materiali del lavoro».

29 K. Marx, Manoscritti economico-filosofici del 1844, cit., p. 14.

${ }^{30}$ Ibidem, p. 64.

${ }^{31}$ Ibidem, p. 65 s.
} 
quale liberazione dal giogo del lavoro coercitivo e salariato, e più in generale dal lavoro tout court - al di là del tema del «lavoro realmente libero» attraversa l'intera produzione di Marx, fino a riemergere, in modo altrettanto chiaro, nel terzo libro del Capitale ove, poco dopo aver ricordato che il pluslavoro, «nel sistema capitalistico come in quello schiavistico ecc., assume semplicemente una forma antagonistica ed è completato dall'ozio assoluto di una parte della società», scrive:

Di fatto, il regno della libertà comincia soltanto là dove cessa il lavoro determinato dalla necessità e dalla finalità esterna; si trova quindi per sua natura oltre la sfera della produzione materiale vera e propria. [...] La libertà in questo campo può consistere soltanto in ciò, che l'uomo socializzato, cioè i produttori associati, regolano razionalmente questo loro ricambio organico con la natura, lo portano sotto il loro comune controllo, invece di essere da esso dominati come da una forza cieca; che essi eseguono il loro compito con il minore possibile impiego di energia e nelle condizioni più adeguate alla loro natura umana e più degne di essa. Ma questo rimane sempre un regno della necessità. $\mathrm{Al}$ di là di esso comincia lo sviluppo delle capacità umane, che è fine a se stesso, il vero regno della libertà, che tuttavia può fiorire soltanto sulle basi di quel regno della necessità. Condizione fondamentale di tutto ciò è la riduzione della giornata lavorativa ${ }^{32}$.

Il framework teorico di Marx mostra così un evidente carico normativo: è ancorato ai principi di libertà ed eguaglianza sostanziali, ai quali si appella, seppur perlopiù implicitamente, per sviluppare la critica del carattere formale della libertà liberale del cittadino, bollata come «immaginaria»e invero ideologica, perché non solo consente il perdurare dell'asservimento dei molti (proletari) da parte di pochi (borghesi), quale si dà nel sistema «schiavista» del lavoro salariato, ma ne offre persino una legittimazione e giustificazione normativa. Tale critica immanente dischiude nel contempo, negandolo, un'alternativa al sistema di oppressione vigente: quella società futura nella quale libertà e uguaglianza saranno realizzate in modo non formale ma sostanziale, anzitutto rispetto alla «schiavitù» del lavoro.

\section{Etica e politica}

È in ragione di questa tematizzazione della società futura che muove anzitutto dalla negazione dell'attuale, e quindi dal privilegiamento sistematico della critica immanente della mancata realizzazione dei principi di uguaglianza e di libertà sanciti sul piano «formale» del diritto borghese, nonché

${ }^{32}$ K. Marx, Il capitale, cit., libro III, sez. VIII, cap. 48, pp. 230-232. 
del loro uso ideologico legittimatorio, che il piano puramente positivo della normatività marxiana mi pare giocare un ruolo secondario nel quadro generale. Tale spiccata propensione è del resto alla base dell'auto-fraintendimento anti-normativistico di Marx. In tal senso, il rilancio del concetto di libertà sociale da parte di Honneth, declinato quale modalità dell'agire etico tale per cui i membri devono giungere ad «aiutarsi reciprocamente nella realizzazione dei reciproci bisogni» ${ }^{33}$, e che rimanda direttamente al «modello dell'amore (Vorbild der Liebe)» positivo proprio delle relazioni persona$\mathrm{li}^{34}$, mi pare quasi rovesciare l'ordine delle priorità teoretico-normative del framework marxiano: come se rischiasse di trasformare in fine - l'accomunazione e l'agire solidale ${ }^{35}$ - quello che in Marx pareva essere piuttosto un mezzo per garantire l'universale autorealizzazione individuale. Risulta allora problematica quella «rotazione prospettica» che ha condotto Honneth a transitare dalla ricostruzione normativa sviluppata nel Diritto della libertà, alla delineazione dei tratti positivi dell'ideale di libertà sociale presentati nella Idea di socialismo, tali da permettere appunto di «dischiudere la possibilità di articolare sul piano istituzionale un ordinamento sociale completamente diverso» ${ }^{36}$. Tale passaggio dal ricostruttivo al costruttivo è così equivalso al privilegiamento del piano propositivo rispetto a quello critico, e quindi a un orientamento che sul piano storico, anche in considerazione del modello etico-solidale di fondo, ricondurrei al socialismo utopistico piuttosto che all'immanentismo normativo marxiano, di taglio prevalentemente negativo.

Inoltre, la valorizzazione dei principi normativi di fraternità e solidarietà, sviluppata alla luce del modello dell'etica della cura e dell'amore personale, ha per certi aspetti esasperato la propensione di Honneth a marginalizzare il ruolo degli interessi, e quindi delle forme in cui gli antagonismi ad essi direttamente correlati vengono tradotti e affrontati nel quadro delle lotte sociali e politiche. Anche quando il tema degli «interessi privati» emerge, con il suo carico problematico e conflittuale, Honneth sembra infatti interpretarlo preferibilmente dalla prospettiva di quella «promessa normativa della solidarietà» immanente alla società moderna che dovrebbe condurre

33 Cfr. per esempio A. Honneth, L'idea di socialismo, cit., p. 40.

34 Ibidem, p. 108.

${ }^{35}$ Cfr. anche ibidem, p. 24: «In tutte queste considerazioni sulle finalità del socialismo, la trasformazione dei mezzi di produzione in proprietà collettiva non viene del resto mai considerata come un fine in se stesso; al contrario, è sempre considerata come una misura necessaria soltanto in quanto mera premessa per la realizzazione di istanze completamente differenti, di natura fondamentalmente morale», e p. 130: «il socialismo deve iniziare a cercare di ottenere l'adesione degli interessati per quel progetto etico che mira a liberare i potenziali presenti nell'ordinamento sociale esistente atti a rinforzare ulteriormente l'essere l'uno-per-l'altro, e volti pertanto alla futura realizzazione della libertà sociale».

${ }^{36}$ Ibidem, p. 10. 
alla risoluzione della sua conflittualità ${ }^{37}$. Da questo punto di vista, mi pare che Honneth prosegua quindi nel percorso che lo ha condotto dall'originaria volontà di integrare sistematicamente il modello utilitaristico giocato sulla categoria di interesse di Marx con la dimensione morale del riconoscimento, al tentativo di rendere quest'ultimo pressoché onnicomprensivo. In Lotta per il riconoscimento, Honneth esplicitava infatti che il suo

modello di conflitto, basato su una teoria del riconoscimento, non può sostituire quel primo modello, di tipo utilitaristico, ma si limita a integrarlo: infatti, fino a che punto un conflitto sociale segua prevalentemente la logica del perseguimento di interessi oppure della reazione morale, rimane sempre una questione empirica ${ }^{38}$.

Ed è precisamente in questo quadro che si collocava la ripresa, preziosa, di quella economia morale, messa in luce da Edward Thompson, che permetteva appunto di rivedere e ampliare in senso normativo, strettamente morale, il framework teorico di matrice marxiana: l'interpretazione delle lotte sociali, anche operaie, oltre alla dimensione degli interessi deve contemplare anche le concezioni della giustizia condivise, nonché le reazioni emotive innescate dalle violazioni delle norme ad esse correlate ${ }^{39}$. Viceversa, a partire perlomeno dal serrato dibattito con Nancy Fraser, Honneth ha vieppiù ampliato i confini del framework elaborato sulla base del concetto morale di riconoscimento, giungendo così a ridurre sempre più lo spazio accordato alla dimensione degli interessi ${ }^{40}$, come mi pare si evinca anche dal privilegiamento del modello etico della cura rilanciato in L'idea di socialismo. Ma anche la rilevanza conferita al processo di «liberazione dalle dipendenze e dalle costrizioni che inibiscono le forme della comunicazione» nella costruzione del futuro socialismo ${ }^{41} \mathrm{mi}$ pare ribadire questa spic-

\footnotetext{
37 Ibidem, p. 87 s.: il socialismo «è il guardiano delle rivendicazioni del "sociale" nel senso forte del termine, in una società in cui la costante riedizione di un'interpretazione unilaterale dei principi fondamentali di legittimazione consente, sotto il manto pretestuoso della libertà individuale, l'affermazione di meri interessi privati, e in tal modo contravviene alla promessa normativa della solidarietà».

${ }^{38}$ Cfr. A. Honneth, Lotta per il riconoscimento. Proposte per un'etica del conflitto (1992), il Saggiatore, Milano 2002, p. 194

39 Cfr. soprattutto E.P. Thompson, L'economia morale delle classi popolari inglesi nel secolo XVIII (1971), et al., Varese 2009; Honneth riprende in tal senso anche i lavori di Barrington Moore $\mathrm{Jr}$, citato anche in L'idea di socialismo, soprattutto la sua idea di «contratto sociale implicito»: cfr. soprattutto $\mathrm{B}$. Moore $\mathrm{Jr}, \mathrm{Le}$ basi sociali dell'obbedienza e della rivolta (1978), Edizioni di Comunità, Milano 1998; ho provato a delineare limiti e prospettive di questa impostazione, insistendo sul ruolo delle emozioni, in M. Solinas, Rivolte mancate. Sulle correlazioni tra emozioni e spregio in Axel Honneth e Barrington Moore Jr, "Paradigmi", XXXV, 2017/2, pp. 207-219.

${ }^{40}$ Cfr. N. Fraser, A. Honneth, Redistribuzione o riconoscimento? Una controversia politico-filosofica, (2003) Meltemi, Roma 2007.
}

${ }^{41}$ A. Honneth, L'idea di socialismo, cit., p. 94 
cata propensione a marginalizzare la sfera degli interessi e dei correlati antagonismi.

Viceversa, uno dei lasciti più preziosi della critica marxiana del capitalismo è stato quello di averci insegnato a soffermare l'attenzione sul ruolo e sulle forme degli interessi che animano i conflitti della società civile, e la cui articolazione gioca spesso un ruolo cruciale nella formazione e nel mantenimento delle relazioni di oppressione e sfruttamento. Certo tali antagonismi non devono più essere letti come se il proletariato avesse degli interessi «obiettivi» già articolati, di cui prima o poi diverrà cosciente, e coincidenti con l'interesse generale della società. Alla luce della rilevanza delle dimensioni normative negate da Marx, i processi di determinazione, auto-percezione e interpretazione dei diversi «interessi» in gioco devono essere fatti oggetto di attenta analisi. In tal senso, si deve allora sviluppare una disamina delle diverse e finanche antitetiche concezioni normative in gioco, schivando ogni naturalismo, e affrontandole piuttosto quali lotte per l'egemonia culturale, aperte e dall'esito sempre incerto. Una via ermeneutica che permette di rilanciare alcuni aspetti cruciali della critica immanente del capitalismo, ad iniziare dalla rilevanza attribuita al principio normativo della libertà sostanziale, intesa anzitutto in relazione alla sfera economica; una forma di critica di cui Marx, al di là dei tanti errori, è stato un maestro. 